Artículo original

\title{
Tendencia de la mortalidad por suicidio en las áreas urbanas y rurales de Colombia, 1979-2014
}

\author{
Pablo Chaparro-Narváez, Diana Díaz-Jiménez, Carlos Castañeda-Orjuela \\ Observatorio Nacional de Salud, Instituto Nacional de Salud, Bogotá, D.C., Colombia
}

Introducción. El suicidio es un grave problema social y de salud pública que afecta a la población de la mayoría de los países del mundo. Se han descrito diferencias en las tasas de suicidio entre las áreas rurales y las urbanas.

Objetivo. Estudiar la tendencia de las tasas de mortalidad por suicidio en Colombia en las áreas de defunción rural y urbana y según sexo, grupo de edad y método de suicidio para el periodo 1979-2014.

Materiales y métodos. Se realizó un estudio ecológico de tendencia temporal a partir de la información de mortalidad del Departamento Administrativo Nacional de Estadística. Se calcularon las tasas de mortalidad específica y ajustada por edad y sexo. Las tendencias de las tasas por área para el periodo de estudio, y tanto por sexo y grupo de edad como por método de suicidio, se estudiaron mediante modelos negativos de regresión binomial y regresión de puntos de inflexión.

Resultados. Un total de 56.448 suicidios se registró en Colombia entre 1979 y 2014 . El riesgo de suicidio fue más alto en el área urbana en hombres, en los grupos etarios de 25 a 44 años y de 65 y más años, y en quienes emplearon el ahorcamiento. El riesgo de suicidio fue mayor en el área rural para los hombres entre los 45 y los 64 años, y para aquellos que recurrieron al disparo de armas de fuego, armas cortantes, ahorcamiento y otros. La tendencia de las tasas en el área urbana mostró su máximo pico en 1999 y, en la rural, en el 2000; posteriormente, en ambas áreas se produjo un descenso paulatino. El ahorcamiento presentó una tendencia al ascenso en hombres en las dos áreas.

Conclusión. El suicidio ha mostrado una tendencia hacia la reducción después del año 2000, con diferencias entre las áreas urbanas y las rurales.

Palabras clave: suicidio; sexo; mortalidad; riesgo relativo; análisis de regresión; Colombia.

The trend in mortality due to suicide in urban and rural areas of Colombia, 1979-2014

Introduction: Suicide is a serious social and public health problem that affects the population in most countries in the world. Differences in suicide rates in rural and urban areas have been previously described.

Recibido: $21 / 03 / 18$

Objective: To study the trend of mortality rates by suicide in Colombia, in rural and urban areas by gender, age group, and suicide method during the years 1979-2014.

Materials and methods: We conducted a temporal trend ecologic study using death certificates from the Departamento Administrativo Nacional de Estadística, DANE. Specific and adjusted by age and gender mortality rates were calculated. We estimated negative

Chaparro-Narváez P, Díaz-Jiménez D, Castañeda-

Orjuela C. Tendencia de la mortalidad por suicidio en las áreas urbanas y rurales de Colombia, 1979-2014 Biomédica. 2019;39:339-53.

https://doi.org/10.7705/biomedica.v39i3.4427

Correspondencia:

Pablo Chaparro-Narváez, Observatorio Nacional de Salud, Instituto Nacional de Salud, Avenida Calle 26 N $51-20$, Bloque B, oficina 208, Bogotá, D.C., Colombia

Teléfono: (571) 2207700 , extensión 1269

pchaparro@ins.gov.co

Contribución de los autores:

Todos los autores participaron en el diseño del estudio, el análisis estadístico, la discusión de resultados y la redacción del manuscrito.

Financiación:

Este trabajo fue realizado con recursos de funcionamiento del Observatorio Nacional de Salud del Instituto Nacional de Salud.

Conflicto de intereses:

Los autores declaran que no existe conflicto de intereses. stratified by gender, age group, and suicide method.

Results: A total of 56,448 suicides was reported in Colombia between 1979 and 2014. The risk of suicide was higher in urban areas for men, individuals between 25 and 44 years, and 65 and over; and for those who used hanging as the suicide method. Also, the risk of suicide was higher in the rural area for men between 45 and 64 years old, and those who used firearms, sharp weapons, hanging, and others as suicide methods. The trend of suicide rates in urban areas showed its maximum peak in 1999 and in the rural ones in 2000 . Then, in the two areas, there was a gradual decrease. Hanging in both areas presented a tendency to rise in men.

Conclusions: Suicide has shown a tendency toward reduction after the year 2000, with differences between urban and rural areas.

Keywords: Suicide; sex; mortality; relative risk (public health); regression analysis; Colombia.

El suicidio es un grave problema social y de salud pública que afecta a la población de la mayoría de los países del mundo. Es un fenómeno en el que intervienen múltiples dinámicas e influyen factores del contexto social, cultural, económico e individual (1). Su definición se hace compleja, pues existen variados conceptos según los distintos puntos de vista desde los cuales se puede examinar. 
En el 2012 el suicidio fue la decimoquinta causa de muerte a nivel mundial, y afectó principalmente a los jóvenes y adultos de edad mediana (2). La Organización Mundial de la Salud (OMS) estimó que ocurrieron 788.000 muertes por suicidio en el mundo en el 2015, para una tasa anual mundial ajustada por edad de 10,7 por 100.000 habitantes (13,6 entre hombres y 7,8 entre mujeres). Para la región de las Américas, la tasa ajustada fue de 9,09 y, junto con la región del Mediterráneo Oriental exhibieron las tasas más bajas por regiones (3).

En Colombia la tasa ajustada fue de 6,2 suicidios por 100.000 habitantes en el 2000 (10,0 entre hombres y 2,7 entre mujeres), y pasó a una de 6,0 en el 2015 (10,1 entre hombres y 2,1 entre mujeres). Según el Observatorio Nacional de Salud, fue la vigesimoprimera causa de muerte (tasa $=5,4 ; I C_{95 \%}$ : $5,2-5,6)$ en 1998 y descendió al vigesimoquinto puesto entre las causas de muerte (tasa=4,3; $\left.I C_{95 \%} 4,1-4,5\right)$ en el 2011 (4).

Según el grado de urbanización, las tasas de suicidio presentan variaciones. Sin embargo, las nociones de áreas urbanas y rurales han sido conceptos que universalmente no se han unificado. En la revisión que Stack publicó en 1982, daba cuenta de las discrepancias que se presentaban en las tasas de suicidio entre las áreas urbanas y las rurales (5); desde entonces, distintos estudios han reportado variaciones y cambios en la tendencia de estas tasas entre dichas áreas (6-13), con resultados controvertidos. Mientras que en unos estudios se han señalado tasas más altas en las áreas rurales (14-16), otros las han reportado en las urbanas (17). En algunas investigaciones en que se consideró el grado de urbanización, se obtuvieron resultados diferentes según el sexo, el grupo de edad, el período de estudio $(11,12,15,18-21)$ y el método de suicidio empleado (22-25).

En cuanto a los métodos de suicidio, estos difieren entre áreas y cambian con el tiempo. Uno de los principales métodos empleado en las áreas rurales ha sido la intoxicación con plaguicidas $(26,27)$, mientras que en las urbanas, se recurre al salto desde un lugar elevado y a la inhalación de monóxido de carbono (28). Las variaciones se han atribuido a las relaciones entre la vida rural y los factores culturales, el aislamiento geográfico e interpersonal, las desigualdades económicas y sociopolíticas que probablemente adquieren su importancia en el momento de la toma de decisiones y, específicamente, las relacionadas con las estrategias para reducir el riesgo (6).

En varios estudios sobre mortalidad por suicidio en el país se han analizado diferentes aspectos desde distintas disciplinas, entre ellos, la tendencia, los factores de riesgo (29-39) y las inequidades (40). No obstante, son escasas las investigaciones que han analizado el suicidio desde el punto de vista de las áreas urbanas y rurales (41). El grado en que las tasas de suicidio varían en respuesta al grado de urbanización, no ha sido completamente investigado.

El objetivo del presente análisis fue evaluar la tendencia de las tasas de mortalidad por suicidio en Colombia en las áreas de defunción urbana y rural, y según sexo, grupo de edad y método de suicidio para el periodo de 1979 a 2014.

\section{Materiales y métodos}

\section{Diseño de estudio}

Se llevó a cabo un estudio ecológico de tendencia temporal para evaluar la tendencia de las tasas de mortalidad por suicidio en Colombia en las áreas 
donde ocurrió la defunción (urbana o rural) entre 1979 y 2014, y según sexo, grupo de edad y método de suicidio.

\section{Fuente de información}

Los datos de muerte por suicidio fueron obtenidos de los certificados individuales de defunción consolidados en las bases de mortalidad del Departamento Administrativo Nacional de Estadística (DANE) (42). Se analizaron las muertes codificadas como suicidio de acuerdo con las normas de la Clasificación Internacional de Enfermedades, novena revisión (CIE-9) (E950-E959) para el periodo de 1979 a 1997 (43) y décima revisión (CIE-10) (X600-X849, Y870) para el periodo 1998-2014 (44). La información sobre la población para el periodo de 1979 a 2014 provino de las proyecciones de población del DANE (45).

\section{Variables de estudio}

Las variables consideradas en el estudio fueron: año de registro de la defunción, número de muertes por año, sexo, edad, área donde ocurrió la defunción, método de suicidio y causa básica de defunción. En el certificado de defunción utilizado en el país, el área donde ocurrió la defunción del fallecido se clasifica en cabecera municipal, centro poblado o rural disperso. Para efectos del estudio, se reagruparon en urbana (cabecera municipal) y rural (centro poblado y rural disperso) (46).

Los diferentes métodos de suicidio se agruparon en ahorcamiento (E953, $X 70$ ), disparo de arma de fuego y material explosivo (E955, X72-X75), envenenamiento (E950-E952, X60-X69), salto desde un lugar elevado (E957, X80), objeto cortante (E956, X78), ahogamiento (E954, X71) y otros mecanismos (E958-X959, X76-X77, X79, X81-X84, Y870).

\section{Análisis estadístico}

Se calcularon las tasas de mortalidad crudas, las tasas ajustadas por edad y sexo según área, las tasas ajustadas por edad según sexo y área, las cuales se estratificaron por grupo de edad y método de suicidio, y las tasas específicas según sexo y área; las tasas se expresaron en muertes por 100.000 habitantes al año. Para el ajuste de las tasas de mortalidad por edad se empleó el método directo, utilizando como referencia la población nacional por grupos de edad quinquenal del censo del 2005.

Las tendencias de las tasas por área según sexo y grupo de edad, y según sexo y método de suicidio, se analizaron por medio de modelos negativos de regresión binomial (47). En la construcción de estos modelos de regresión, la variable independiente fue el año para la tasa total y, para los demás modelos de regresión, las variables explicativas fueron el año y el sexo. Se calculó la razón de prevalencia (RP) y su intervalo de confianza del $95 \%\left(\mathrm{IC}_{95 \%}\right)$ junto con su valor de $\mathrm{p}$.

La tendencia de las tasas de mortalidad por área según sexo, grupo de edad y método del suicidio, se determinó mediante el análisis de regresión de puntos de inflexión (joinpoint Poisson), procedimiento que empleó las tasas ajustadas por edad. Este análisis se utiliza para establecer el momento en que se producen cambios significativos en la tendencia, y estima la magnitud del aumento o la disminución observados en cada intervalo mediante el porcentaje de cambio anual. 
El análisis comienza con una cantidad mínima de puntos de inflexión, y prueba si uno o más puntos son estadísticamente significativos y se deben agregar al modelo. El número de puntos se determina por medio de pruebas de permutación, cada una de las cuales tiene un nivel de significación asintótico correcto. Este nivel de significación se encuentra usando métodos de Montecarlo (48). El modelo final muestra los puntos de inflexión que mejor se adaptan cuando la tasa cambia significativamente.

En este análisis se calculó el porcentaje de cambio anual (PCA) y el porcentaje promedio de cambio anual. Con el primero, se caracterizaron las tendencias observadas de la siguiente forma: con PCA $>0$ y significación estadística se interpretó un aumento en la tendencia y con $\mathrm{PCA}<0$ y significación estadística, una reducción; con un cambio menor o igual a 0,5\% anual $(-0,5 \leq \mathrm{PCA} \leq 0,5 \%)$ y significación estadística, se interpretó como estable, y con un cambio mayor de $0,5 \%$ anual (PCA $<-0,5 \%$ o PCA $>0,5 \%$ ) pero sin significación estadística, se juzgó sin cambios significativos (49).

Para el procesamiento de la información obtenida se emplearon hojas de cálculo de Microsoft Excel ${ }^{\mathrm{TM}}$. La información se analizó con los programas Stata $^{\mathrm{TM}}$, versión 12, y Joinpoint Regression ${ }^{\mathrm{TM}}$ (50).

\section{Resultados}

\section{Tasas de suicidio}

Un total de 56.448 suicidios se registraron en Colombia entre 1979 y 2014. El 74,2\% (41.890) ocurrió en el área urbana $(p<0,05)$. Las tasas promedio de mortalidad para el periodo fueron de 4,2 suicidios por 100.000 habitantes para el área urbana, y de 3,7 para el área rural.

Por sexo, la tasa promedio fue de 6,7 para los hombres del área urbana y de 5,9 para los del área rural; para las mujeres del área urbana, la tasa promedio fue de 1,8 y, para las del área rural, de 1,6.

Por grupos de edad, en los hombres las tasas fueron más altas en el grupo de 65 y más años y en el de 15 a 24 años, en tanto que en las mujeres las tasas más altas de evidenciaron en el grupo de 15 a 24 años.

Por método de suicidio, las tasas más altas en hombres fueron las de ahorcamiento (área urbana: 2,37; área rural: 1,65), seguidas por las de disparo de armas de fuego (área urbana: 2,25; área rural: 2,32); en las mujeres, fueron las de envenenamiento (área urbana: 0,95; área rural: 0,90), seguidas de las de ahorcamiento (área urbana: 0,42; área rural: 0,32).

\section{Modelos de regresión}

El riesgo general de suicidio para los hombres de las áreas urbanas o rurales fue cuatro veces mayor con respecto a las mujeres. En el área urbana el riesgo de suicidio fue más alto en hombres de los grupos etarios de 25 a 44 años de edad y de 65 y más años, y para los que recurrieron al ahorcamiento. En el área rural el riesgo de suicidio fue más alto para hombres, para el grupo etario de 45 a 64 años de edad, y para los que recurrieron al ahogamiento, el disparo con armas de fuego y el uso de armas cortantes (cuadro 1).

El modelo negativo de regresión binomial para los datos anuales totales reveló una tendencia anual creciente en las tasas de suicidio durante el período de estudio (cuadro 1). Este modelo mostró que por sexo y grupos de 
Cuadro 1. Suicidio: estadística descriptiva y regresión binomial negativa según el área en que ocurrió, Colombia, 1979-2014

\begin{tabular}{|c|c|c|c|c|c|c|c|c|c|c|c|c|c|}
\hline \multirow[b]{3}{*}{ Total } & \multirow{3}{*}{ Sexo } & \multicolumn{5}{|c|}{ Área urbana } & \multicolumn{6}{|c|}{ Área rural } & \multirow{3}{*}{$\frac{\mathbf{p}}{<0,001}$} \\
\hline & & \multirow{2}{*}{$\frac{n}{41.890}$} & \multirow{2}{*}{$\begin{array}{c}\text { Tasa } \\
4,2\end{array}$} & \multirow{2}{*}{$\begin{array}{c}\mathbf{R P} \\
1,02\end{array}$} & \multicolumn{2}{|c|}{$\mathrm{IC}_{95} \%$} & \multirow{2}{*}{$\frac{\mathbf{p}}{<0,001}$} & \multirow{2}{*}{$\frac{\mathbf{n}}{13.989}$} & \multirow{2}{*}{$\begin{array}{c}\text { Tasa } \\
3,7\end{array}$} & \multirow{2}{*}{$\begin{array}{c}\mathbf{R P} \\
1,02\end{array}$} & \multicolumn{2}{|c|}{$\mathrm{IC}_{95} \%$} & \\
\hline & & & & & 1,02 & 1,03 & & & & & 1,02 & 1,03 & \\
\hline Total, por sexo & Femenino & 9.348 & 1,8 & & & & & 2.731 & 1,6 & & & & \\
\hline \multicolumn{14}{|l|}{$\begin{array}{l}\text { Grupos de edad } \\
\text { (años) }\end{array}$} \\
\hline \multirow[t]{2}{*}{15 a 24} & Masculino & 9.827 & 10,8 & 2,35 & 0,56 & 9,76 & 0,24 & 3.566 & 9,5 & 2,27 & 0,54 & 9,45 & 0,26 \\
\hline & Femenino & 4.355 & 4,6 & & & & & 1.396 & 4,2 & & & & \\
\hline \multirow[t]{2}{*}{25 a 44} & Masculino & 12.887 & 9,4 & 5,00 & 1,45 & 17,22 & 0,01 & 4.210 & 8,6 & 4,92 & 1,38 & 17,50 & 0,01 \\
\hline & Femenino & 2.860 & 1,9 & & & & & 772 & 1,8 & & & & \\
\hline 45 a 64 & Masculino & 5.926 & 8,3 & 6,40 & 2,30 & 17,79 & $<0,001$ & 2.123 & 7,6 & 7,22 & 2,46 & 21,25 & $<0,001$ \\
\hline \multicolumn{14}{|l|}{$\begin{array}{l}\text { Métodos de } \\
\text { suicidio }\end{array}$} \\
\hline \multirow[t]{2}{*}{ Ahorcamiento } & Masculino & 11.402 & 2,37 & 6,80 & 3,04 & 15,25 & $<0,001$ & 3.116 & 1,65 & 5,95 & 2,46 & 14,41 & $<0,001$ \\
\hline & Femenino & 2.164 & 0,42 & & & & & 541 & 0,32 & & & & \\
\hline \multirow[t]{2}{*}{ Ahogamiento } & Masculino & 224 & 0,05 & 4,10 & 1,15 & 14,68 & 0,03 & 233 & 0,12 & 5,95 & 2,46 & 14,41 & $<0,001$ \\
\hline & Femenino & 66 & 0,01 & & & & & 86 & 0,05 & & & & \\
\hline \multirow{2}{*}{$\begin{array}{l}\text { Saltar desde un } \\
\text { lugar elevado }\end{array}$} & Masculino & 1.213 & 0,25 & 3,33 & 0,94 & 11,81 & 0,06 & 115 & 0,06 & 4,21 & 0,98 & 18,18 & 0,05 \\
\hline & Femenino & 493 & 0,09 & & & & & 28 & 0,02 & & & & \\
\hline \multirow[t]{2}{*}{ Otros } & Masculino & 811 & 0,17 & 3,16 & 1,00 & 9,99 & 0,05 & 382 & 0,2 & 4,19 & 1,47 & 11,91 & 0,01 \\
\hline & Femenino & 322 & 0,06 & & & & & 87 & 0,05 & & & & \\
\hline Envenenamiento & Masculino & 7.082 & 1,46 & 1,77 & 0,59 & 5,34 & 0,31 & 2.766 & 1,47 & 1,73 & 0,55 & 5,49 & 0,35 \\
\hline
\end{tabular}

Fuente: Estadísticas vitales, DANE

$\mathrm{n}$ : número para cada estrato; RP: razón de prevalencia

edad, las tasas fueron mayores en el área rural, a excepción del grupo de 65 y más años; y según el método de suicidio, estas fueron mayores en el área urbana que en la rural (cuadro 2).

En el área urbana, el análisis de regresión de puntos de inflexión mostró su máximo pico en hombres en 1999; entre 1979 y 1999 hubo una tendencia significativamente creciente, seguida de un descenso significativo hasta el 2014; en el área rural el comportamiento fue similar, y el máximo pico se presentó en el 2000 (figura 1).

Para el periodo 1979-2014, por sexo y grupos de edad, el porcentaje promedio de cambio anual del análisis de regresión de puntos de inflexión indicó un aumento significativo del suicidio en hombres de los grupos de 5 a 14 años de edad del área urbana, de 0 a 14 años y de 65 y más años, en el área rural, así como un aumento significativo en mujeres de 45 a 64 años de edad del área urbana. El modelo presentó ajustes significativos en su tendencia en los distintos segmentos para cada uno de los grupos de edad, excepto para las mujeres del área urbana de 65 y más años (figura 1, cuadro 3). 
Cuadro 2. Suicidio según área, regresión binomial negativa, Colombia, 1979-2014

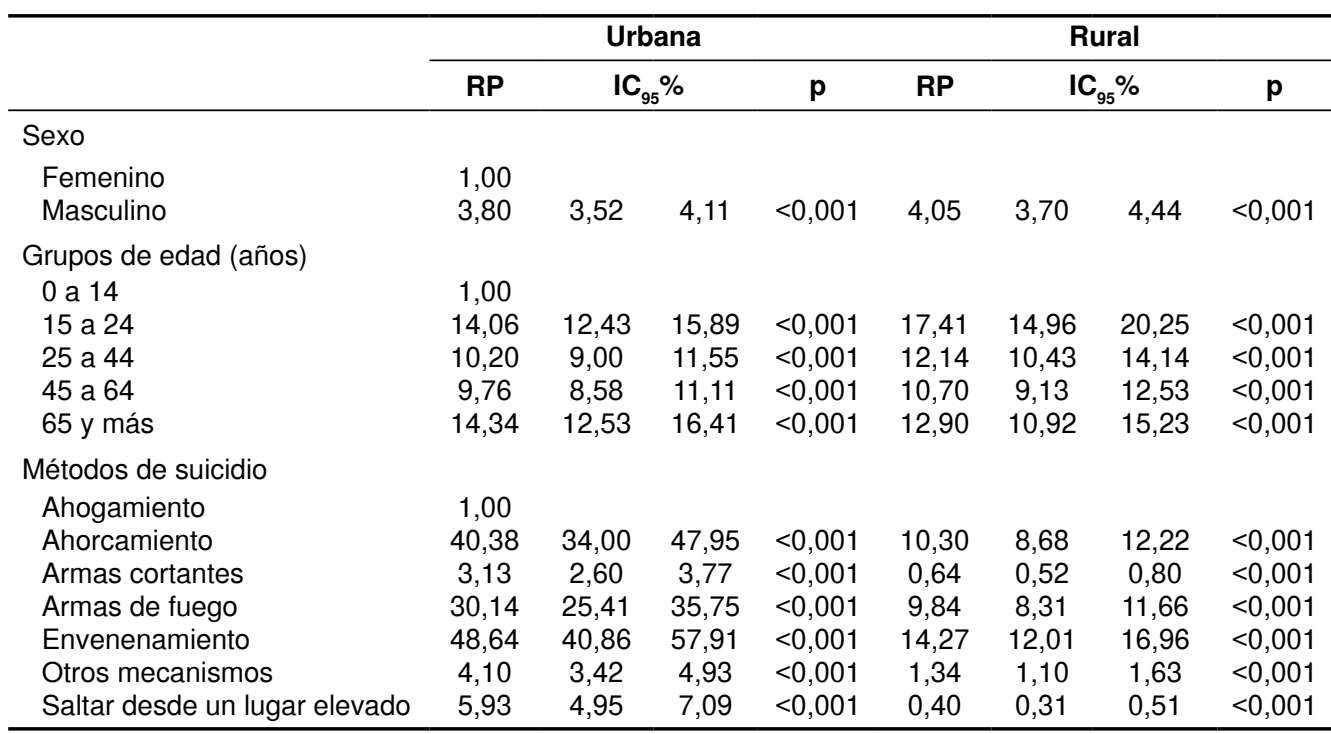

Fuente: Estadísticas vitales, DANE

RP: razón de prevalencia
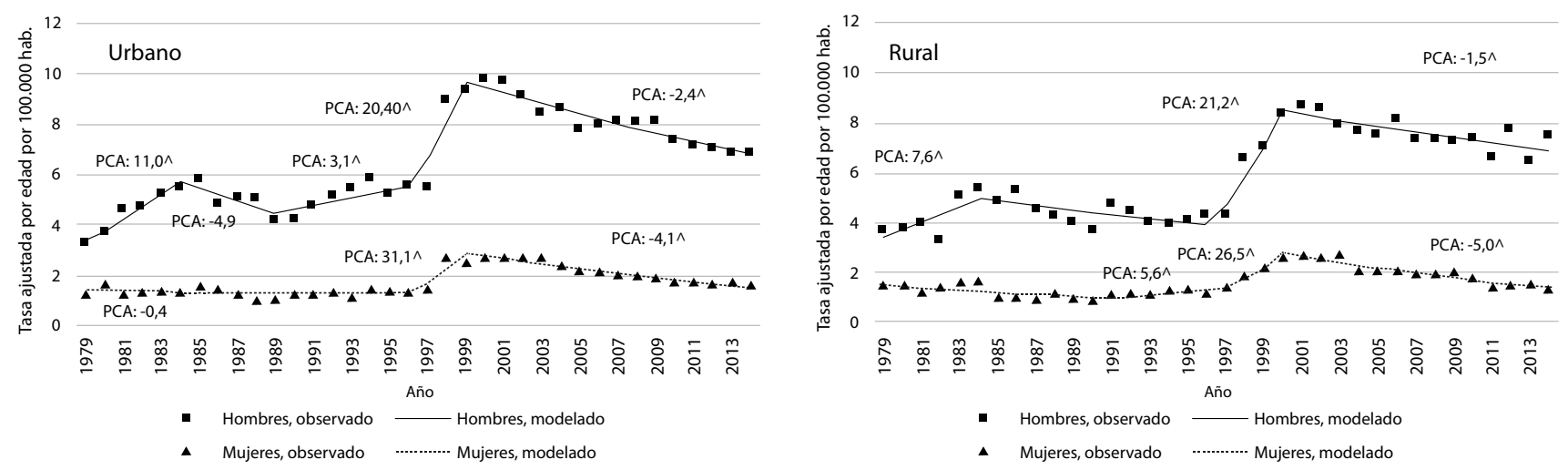

Figura 1. Tendencia de las tasas de mortalidad por suicidio ajustadas por edad, sexo y área, Colombia, 1979-2014

PCA: porcentaje de cambio anual

$\wedge^{\wedge}$ El PCA es significativamente distinto de $0(p<0,05)$.

Fuente: Estadísticas vitales, DANE

Para todo el periodo de estudio en el área urbana, el porcentaje promedio de cambio anual del análisis de regresión de puntos de inflexión por método de suicidio indicó un aumento significativo del suicidio en hombres por ahorcamiento y ahogamiento, y en mujeres, por ahorcamiento y salto desde un lugar elevado; además, un descenso significativo en hombres por otros mecanismos y los no especificados, y en mujeres, por armas de fuego, otros mecanismos y los no especificados.

En el área rural se evidenció un aumento significativo del suicidio en hombres por ahorcamiento y ahogamiento, así como un descenso significativo en hombres por otros mecanismos, los no especificados y armas cortantes, y en mujeres, por armas de fuego. El modelo presentó ajustes significativos en la tendencia en los distintos segmentos para cada uno de los métodos de suicidio, excepto para las mujeres del área rural que recurrieron al ahorcamiento (figura 2, cuadro 4). 
Cuadro 3. Tendencias de las tasas de suicidio por grupo de edad según área de defunción y sexo, y porcentaje de cambio anual de la mortalidad por suicidio, Colombia, 1979-2014

\begin{tabular}{|c|c|c|c|c|c|c|c|c|}
\hline \multicolumn{9}{|c|}{ Área urbana } \\
\hline \multirow[b]{2}{*}{$\begin{array}{l}\text { Grupo de } \\
\text { edad (años) }\end{array}$} & \multicolumn{4}{|c|}{ Hombres } & \multicolumn{4}{|c|}{ Mujeres } \\
\hline & Periodo & PCA & Tendencia & PPCA & Periodo & PCA & Tendencia & PPCA \\
\hline \multirow[t]{3}{*}{0 a 14} & $1979-2008$ & $6,0^{\wedge}(4,7-7,4)$ & Aumento & \multirow{3}{*}{$3,6^{\wedge}(1,7$ a 5,4$)$} & $1979-1995$ & $-4,0^{\wedge}(-7,4$ a $-0,5)$ & Reducción & \multirow{3}{*}{$1,5(-5,0$ a 8,5$)$} \\
\hline & 2008-2014 & $-7,4(-15,3$ a 1,2$)$ & Sin cambios & & 1995-1998 & $70,6(-22,2$ a 274,0$)$ & Sin cambios & \\
\hline & & & & & $1998-2014$ & $-2,6^{\wedge}(-4,6$ a $-0,6)$ & Reducción & \\
\hline \multirow[t]{4}{*}{$15-24$} & $1979-1996$ & $-0,4(-0,8$ a 1,6$)$ & Sin cambios & & $1979-1993$ & $-3,5^{\wedge}(-5,8 a-1,2)$ & Reducción & \multirow{4}{*}{$-1,1(-2,8$ a 0,5$)$} \\
\hline & $1996-2000$ & $21,8^{\wedge}(6,5$ a 39,3$)$ & Aumento & \multirow{3}{*}{$0,7(-1,7$ a 3,2$)$} & $1993-2001$ & $14,6^{\wedge}(8,5$ a 21,1$)$ & Aumento & \\
\hline & $2000-2003$ & $-10,5(-28,4$ a 11,8$)$ & Sin cambios & & 2001-2014 & $-7,3^{\wedge}(-9,1$ a $-5,5)$ & Reducción & \\
\hline & $2003-2014$ & $-2,4^{\wedge}(-4,0$ a $-0,8)$ & Reducción & & & & & \\
\hline \multirow[t]{3}{*}{$25-44$} & 1979-1996 & $1,1(-0,1$ a 2,4$)$ & Sin cambios & \multirow{3}{*}{$1,4(-0,9$ a 3,6$)$} & $1979-1995$ & $-1,0(-0,6$ a 2,6$)$ & Sin cambios & \multirow{3}{*}{$1,0(-1,9$ a 3,9$)$} \\
\hline & 1996-1999 & $20,4(-7,0$ a 55,9$)$ & Sin cambios & & $1995-1998$ & $19,0(-14,9$ a 66,5$)$ & Sin cambios & \\
\hline & $1999-2014$ & $-1,8^{\wedge}(-2,7$ a $-0,9)$ & Reducción & & $1998-2014$ & $-2,1^{\wedge}(-3,1$ a $-1,1)$ & Reducción & \\
\hline \multirow[t]{4}{*}{$45-64$} & $1979-1985$ & $13,8^{\wedge}(6,1$ a 22,1$)$ & Aumento & & $1979-2014$ & $1,8^{\wedge}(1,0$ a 2,7$)$ & Aumento & \multirow[t]{4}{*}{$1,8^{\wedge}(1,0$ a 2,7$)$} \\
\hline & $1985-1990$ & $-10,4(-20,9$ a 1,5$)$ & Sin cambios & \multirow{3}{*}{$2,3(-0,0$ a 4,6$)$} & & & & \\
\hline & 1990-1999 & $10,1^{\wedge}(6,2$ a 14,2$)$ & Aumento & & & & & \\
\hline & $1999-2014$ & $-2,0^{\wedge}(-3,0 \mathrm{a}-1,1)$ & Reducción & & & & & \\
\hline \multirow[t]{3}{*}{65 y más } & $1979-1996$ & $3,0^{\wedge}(1,0$ a 5,0$)$ & Aumento & \multirow[t]{3}{*}{$1,5(-1,9$ a 4,9$)$} & $1979-2014$ & $0,1(-1,1$ a 1,4$)$ & Sin cambios & \multirow[t]{3}{*}{$0,1(-1,1$ a 1,4$)$} \\
\hline & 1996-1999 & $14,3(-22,4$ a 68,3$)$ & Sin cambios & & & & & \\
\hline & $1999-2014$ & $-2,5^{\wedge}(-3,9 a-1,2)$ & Reducción & & & & & \\
\hline \multicolumn{9}{|c|}{ Área rural } \\
\hline \multirow[t]{3}{*}{0 a 14} & $1979-2014$ & $4,5^{\wedge}(3,1$ a 5,8$)$ & & \multirow[t]{3}{*}{$4,5^{\wedge}(3,1$ a 5,8$)$} & $1979-1995$ & $-7,0^{\wedge}(-12,6 a-1,1)$ & Reducción & \multirow{3}{*}{$0,5(-6,5$ a 8,0$)$} \\
\hline & & & Aumento & & 1995-1999 & $62,9(-9,7$ a 193,8$)$ & Sin cambios & \\
\hline & & & & & $1999-2014$ & $-4,1^{\wedge}(-7,8$ a $-0,2)$ & Reducción & \\
\hline \multirow[t]{5}{*}{$15-24$} & $1979-1996$ & $-0,1(-2,2$ a 2,1$)$ & Sin cambios & & $1979-1983$ & $8,4(-1,8$ a 19,7$)$ & Sin cambios & \multirow{5}{*}{$0,2(-2,4$ a 2,8$)$} \\
\hline & $1996-2000$ & $19,8(-6,2$ a 53,1$)$ & Sin cambios & & $1983-1988$ & $-14,0^{\wedge}(-21,5$ a $-5,7)$ & Reducción & \\
\hline & $2000-2014$ & $-2,9^{\wedge}(-4,7$ a $-1,0)$ & Reducción & $0,9(-2,1$ a 3,9$)$ & $1988-1997$ & $6,1^{\wedge}(2,7$ a 9,7$)$ & Aumento & \\
\hline & & & & & $1997-2000$ & $30,4^{\wedge}(4,6$ a 62,6$)$ & Aumento & \\
\hline & & & & & $2000-2014$ & $-5,8^{\wedge}(-6,8$ a $-4,8)$ & Reducción & \\
\hline \multirow[t]{3}{*}{$25-44$} & $1979-1997$ & $0,4(-1,1$ a 1,9$)$ & Sin cambios & & $1979-2003$ & $3,7^{\wedge}(2,1$ a 5,3$)$ & Aumento & \multirow{3}{*}{$0,6(-1,1$ a 2,4$)$} \\
\hline & $1997-2000$ & $23,2(-11,4$ a 71,3$)$ & Sin cambios & $1,4(-1,5$ a 4,3$)$ & 2003-2014 & $-5,8^{\wedge}(-10,2 a-1,1)$ & Reducción & \\
\hline & $2000-2014$ & $-1,5^{\wedge}(-2,9 a-0,1)$ & Reducción & & & & & \\
\hline $45-64$ & $1979-1996$ & $-2,1^{\wedge}(-3,7$ a $-0,4)$ & Reducción & & $1979-1987$ & $-11,3(-23,4$ a 2,6$)$ & Sin cambios & $-02(-36$ - 35$)$ \\
\hline & $1996-2000$ & $20,5(-1,5$ a 47,4$)$ & Sin cambios & $1,0(-1,4$ a 3,5$)$ & $1987-2014$ & $3,4^{\wedge}(1,3$ a 5,6$)$ & Aumento & $0,2, \quad(0,0$ a $u, v)$ \\
\hline & $2000-2014$ & $-0,2(-1,6$ a 1,2$)$ & Sin cambios & & & & & \\
\hline 65 y más & $1979-2014$ & $2,3^{\wedge}(1,5$ a 3,1$)$ & Aumento & $2,3^{\wedge}(1,5$ a 3,1$)$ & & & & \\
\hline
\end{tabular}

PCA: porcentaje de cambio anual; PPCA: porcentaje promedio de cambio anual

$\wedge$ El PCA es significativamente distinto de $0(p<0,05)$.

Fuente: Estadísticas vitales, DANE

\section{Discusión}

\section{Principales resultados}

En este estudio se encontraron diferencias significativas en las tasas de suicidio entre las áreas urbanas y las rurales según el sexo, el grupo de edad y el método empleado. Las tasas de suicidio en ambas áreas fueron más altas en hombres. Entre los hombres, las tasas fueron más altas en el área urbana, en tanto que entre las mujeres, las tasas por áreas fueron similares. 
Cuadro 4. Tendencias de las tasas de mortalidad según los métodos de suicidio, el área de defunción y el sexo, Colombia, 1979-2014

\begin{tabular}{|c|c|c|c|c|c|c|c|c|}
\hline \multicolumn{9}{|c|}{ Área urbana } \\
\hline \multirow{2}{*}{ Características } & \multicolumn{4}{|c|}{ Hombres } & \multicolumn{4}{|c|}{ Mujeres } \\
\hline & Periodo & PCA & Tendencia & PPCA & Periodo & PCA & Tendencia & PPCA \\
\hline \multirow[t]{3}{*}{ Ahorcamiento } & $1979-1996$ & $5,4^{\wedge}(3,3$ a 7,6$)$ & Aumento & \multirow{3}{*}{$5,5^{\wedge}(2,2$ a 9,1$)$} & 1979-2004 & $9,2^{\wedge}(7,5$ a 10,9$)$ & Aumento & \multirow{3}{*}{$6,8^{\wedge}(5,3$ a 8,2$)$} \\
\hline & 1996-1999 & $25,8(-13,7$ a 83,4$)$ & Sin cambios & & 2004-2014 & $0,9(-2,0$ a 3,9$)$ & Sin cambios & \\
\hline & $1999-2014$ & $2,0^{\wedge}(1,0$ a 3,1$)$ & Aumento & & & & & \\
\hline \multirow[t]{3}{*}{ Envenenamiento } & 1979-1995 & $0,5(-0,9$ a 1,9$)$ & Estable & \multirow{3}{*}{$0,2(-1,4$ a 1,8$)$} & 1979-1992 & $-4,3^{\wedge}(-6,8$ a $-1,6)$ & Reducción & \multirow{3}{*}{$-1,2(-2,9$ a 0,4$)$} \\
\hline & $1995-1999$ & $23,7^{\wedge}(8,5$ a 41,0$)$ & Aumento & & $1992-2000$ & $15,7^{\wedge}(9,7$ a 22,1$)$ & Aumento & \\
\hline & $1999-2014$ & $-5,6^{\wedge}(-6,6$ a $-4,6)$ & Reducción & & $2000-2014$ & $-7,2^{\wedge}(-8,7$ a $-5,6)$ & Reducción & \\
\hline \multirow[t]{4}{*}{ Armas de fuego } & $1979-1983$ & $14,8^{\wedge}(5,2$ a 25,2$)$ & Aumento & & 1979-2002 & $1,3^{\wedge}(0,0$ a 2,7$)$ & Aumento & \\
\hline & 1983-1996 & $-1,8^{\wedge}(-3,1$ a $-0,4)$ & Reducción & \multirow{3}{*}{$-0,5(-2,0$ a 1,0$)$} & $2002-2014$ & $-12,0^{\wedge}(-15,8$ a $-8,1)$ & Reducción & $-3,5^{\wedge}(-5,0$ a $-1,9)$ \\
\hline & $1996-2000$ & $13,9^{\wedge}(3,3$ a 25,5$)$ & Aumento & & & & & \\
\hline & $2000-2014$ & $-7,0^{\wedge}(-7,9$ a $-6,1)$ & Reducción & & & & & \\
\hline \multirow{4}{*}{$\begin{array}{l}\text { Saltar desde un } \\
\text { lugar elevado }\end{array}$} & 1979-1985 & $19,4^{\wedge}(4,5$ a 36,5$)$ & Aumento & \multirow{3}{*}{$3,2(-1,3$ a 8,0$)$} & 1979-2014 & $1,6^{\wedge}(0,3$ a 2,9$)$ & Aumento & \multirow[t]{4}{*}{$1,6^{\wedge}(0,3$ a 2,9$)$} \\
\hline & $1985-1990$ & $-22,4(-40,0$ a 0,2$)$ & Sin cambios & & & & & \\
\hline & 1990-1999 & $19,7^{\wedge}(11,6$ a 28,4$)$ & Reducción & & & & & \\
\hline & $1999-2014$ & $-2,0^{\wedge}(-3,5$ a $-0,4)$ & Reducción & & & & & \\
\hline \multirow{2}{*}{$\begin{array}{l}\text { Otros mecanismos y } \\
\text { los no especificados }\end{array}$} & $1979-1993$ & $0,9(-2,2$ a 4,1$)$ & Sin cambios & \multirow{4}{*}{$-2,6^{\wedge}(-4,1$ a $-1,1)$} & 1979-2014 & $-2,7^{\wedge}(-4,1$ a $-1,4)$ & Reducción & \multirow[t]{4}{*}{$-2,7^{\wedge}(-4,1$ a $-1,4)$} \\
\hline & $1993-2014$ & $-4,8^{\wedge}(-6,5$ a $-3,2)$ & Reducción & & & & & \\
\hline Armas cortantes & $1979-2014$ & $-0,2(-1,0$ a 0,7$)$ & Sin cambios & & & & & \\
\hline Ahogamiento & $1979-2014$ & $1,6^{\wedge}(0,1$ a 3,0$)$ & Aumento & & & & & \\
\hline \multicolumn{9}{|c|}{ Área rural } \\
\hline \multirow[t]{3}{*}{ Ahorcamiento } & 1979-1996 & $1,0(-0,9$ a 3,0$)$ & Sin cambios & \multirow{3}{*}{$5,2^{\wedge}(1,8$ a 8,7$)$} & 1979-1995 & $1,6(-2,7$ a 6,1$)$ & Sin cambios & \multirow{3}{*}{$4,4(-0,5$ a 9,6$)$} \\
\hline & 1996-1999 & $41,6(-3,1$ a 107,2$)$ & Sin cambios & & 1995-1999 & $28,3(-13,7$ a 90,8$)$ & Sin cambios & \\
\hline & $1999-2014$ & $3,8^{\wedge}(2,8$ a 4,9$)$ & Aumento & & $1999-2014$ & $1,8(-0,5$ a 4,2$)$ & Sin cambios & \\
\hline \multirow[t]{3}{*}{ Envenenamiento } & $1979-1996$ & $-1,2(-3,2$ a 0,9$)$ & Sin cambios & \multirow{3}{*}{$0,4(-2,4$ a 3,2$)$} & $1979-1990$ & $-6,6^{\wedge}(-10,3$ a $-2,9)$ & Reducción & \multirow[t]{3}{*}{$-1,5(-3,2$ a 0,3$)$} \\
\hline & $1996-2000$ & $30,3^{\wedge}(3,3$ a 64,4$)$ & Aumento & & 1990-2002 & $11,8^{\wedge}(8,4$ a 15,3$)$ & Aumento & \\
\hline & $2000-2014$ & $-5,1^{\wedge}(-6,8$ a $-3,3)$ & Reducción & & 2002-2014 & $-8,8^{\wedge}(-11,2 a-6,3)$ & Reducción & \\
\hline \multirow{4}{*}{$\begin{array}{l}\text { Armas de fuego y } \\
\text { explosivos }\end{array}$} & $1979-1983$ & $16,2^{\wedge}(2,5$ a 31,8$)$ & Aumento & & 1979-2002 & $0,5(-1,3$ a 2,3$)$ & Estable & $-2,1^{\wedge}(-4,1$ a $-0,0)$ \\
\hline & 1983-1997 & $-1,7^{\wedge}(-3,1$ a $-0,2)$ & Reducción & $1,2(-1,4$ a 3.9 & 2002-2014 & $-6,8^{\wedge}(-11,6 a-1,7)$ & Reducción & \\
\hline & $1997-2000$ & $20,3(-7,3$ a 56,1$)$ & Sin cambios & & & & & \\
\hline & $2000-2014$ & $-3,5^{\wedge}(-4,7$ a $-2,4)$ & Reducción & & & & & \\
\hline $\begin{array}{l}\text { Saltar desde un } \\
\text { lugar elevado }\end{array}$ & & & & & & & & \\
\hline Otros mecanismos y & 1979-1984 & $10,9(-4,7$ a 29,0$)$ & Sin cambios & $-3,2^{\wedge}(-5,4$ a $-0,9)$ & & & & \\
\hline 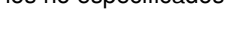 & 1984-2014 & $-5,4^{\wedge}(-6,6$ a $-4,2)$ & Reducción & & & & & \\
\hline Armas cortantes & 1979-2014 & $-1,6^{\wedge}(-2,8$ a $-0,4)$ & Reducción & $-1,6^{\wedge}(-2,8$ a $-0,4)$ & & & & \\
\hline Ahogamiento & $1979-2014$ & $1,9^{\wedge}(0,4$ a 3,4$)$ & Aumento & $1,9^{\wedge}(0,4$ a 3,4$)$ & & & & \\
\hline
\end{tabular}

PCA: porcentaje de cambio anual; PPCA: porcentaje promedio de cambio anual

$\wedge$ El PCA es significativamente distinto de $0(p<0,05)$.

Fuente: Estadísticas vitales, DANE

En hombres y mujeres del área urbana se observó que las tasas presentaron una tendencia decreciente significativa, con un punto de inflexión en 1999. En hombres y mujeres del área rural se observaron patrones de cambio en las tasas similares a los del área urbana.

Según el método de suicidio empleado, en los hombres las tasas de ahorcamiento se incrementaron en las dos áreas, predominando las del área urbana; las tasas debidas a disparo de armas de fuego disminuyeron, especialmente en el área rural; las tasas de envenenamiento en el área 
Área urbana
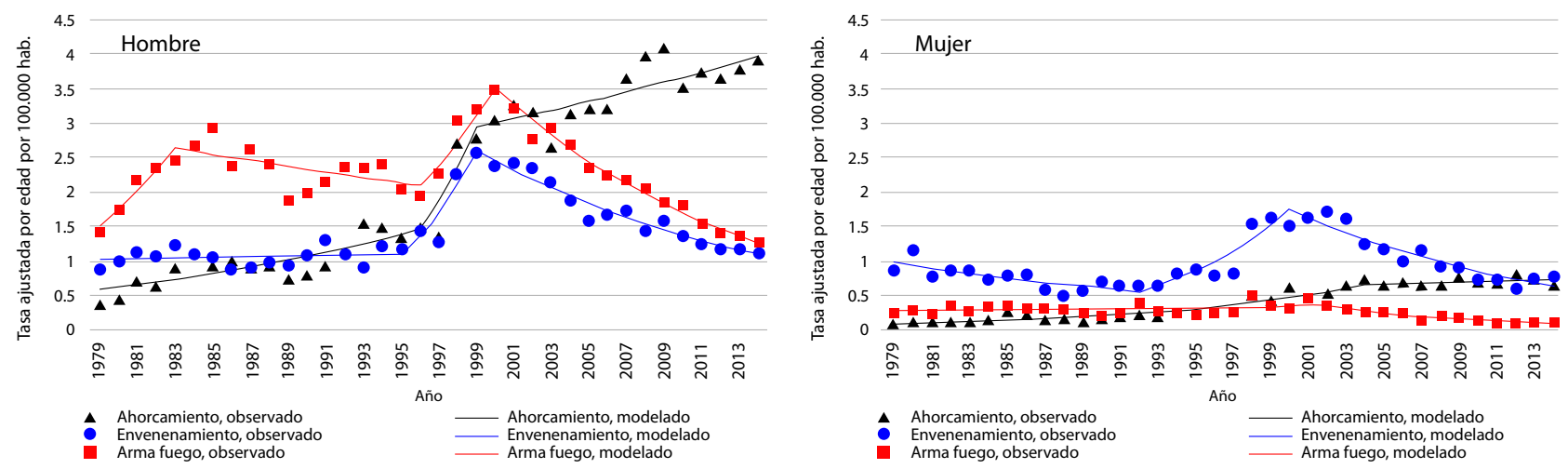

Área rural
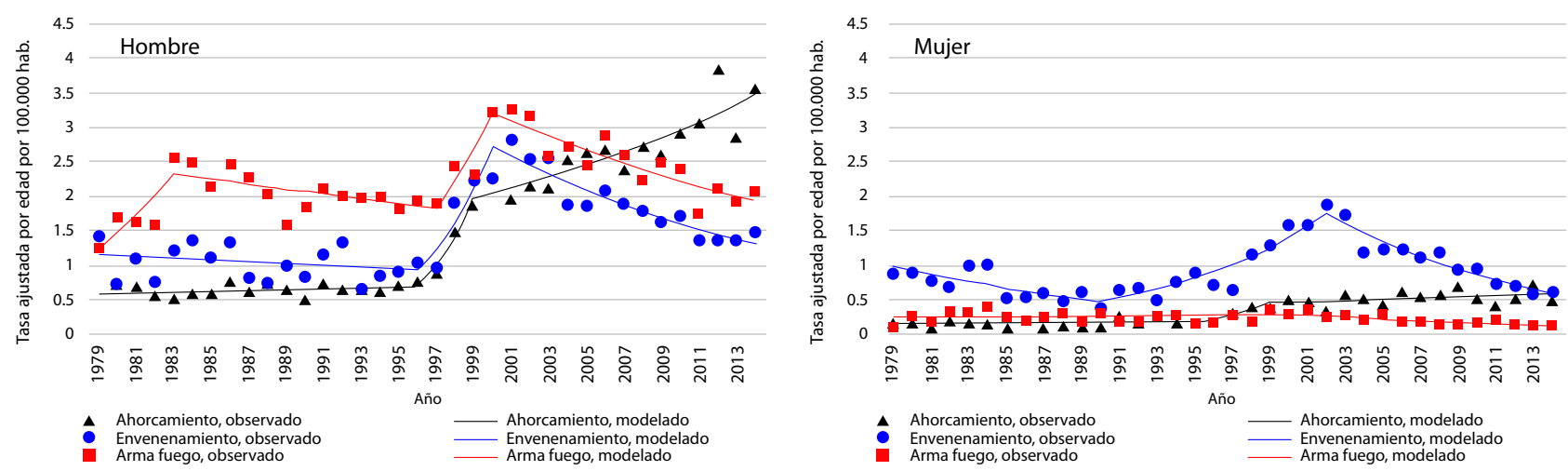

Figura 2. Tendencia de las tasas de mortalidad por suicidio ajustadas por edad, sexo y área, Colombia, 1979-2014 Fuente: Estadísticas vitales, DANE

urbana presentaron una disminución mayor. En las mujeres del área rural las tasas por ahorcamiento presentaron un mayor incremento, mientras que las tasas por envenenamiento fueron similares.

\section{Comparación con otros estudios}

Al igual que lo encontrado en Corea (12), las tasas de suicidio para los hombres fueron tres veces mayores que para las mujeres, tanto en el área urbana como en el área rural. Asimismo, en muchos estudios en los que no se analizan las tasas de suicidio por áreas urbanas y rurales, se ha observado que las tasas son más altas en los hombres. Se ha indicado que esta situación puede estar relacionada con las dificultades económicas, las responsabilidades sociales, la elección de métodos más letales (51) y el abuso en el consumo de alcohol (52).

También, como en Corea (12), las tasas de suicidio aumentaron con la edad y la tasa del grupo de edad de 65 y más años fue diez veces más alta en las áreas urbanas que en las rurales. Se ha sugerido que el desempleo tiene una influencia positiva en los suicidios, y varía por grupos de edad y por sexo. Los hombres en edad productiva son particularmente sensibles (53); al reducir los ingresos y producir incertidumbre sobre los ingresos futuros, el desempleo aumenta la probabilidad de suicidio, efecto que es más fuerte en individuos de mediana edad (54). Entre los factores ligados al suicidio en los ancianos se han sugerido la depresión, el estado de salud (enfermedades 
crónicas, hospitalizaciones repetidas), la familia (pérdida de seres queridos), y los sociales y ambientales (jubilación, pérdida de prestigio, rechazo de la sociedad, competencia con generaciones más jóvenes) (55).

De igual forma, las tasas de suicidio fueron mayores en las áreas urbanas como se ha reportado en los estudios del suroeste de Irán, entre 2004 y 2009 (17), y en Australia y Beijing, entre 1991 y 1996 (56).

En diversos estudios se han relacionado las variaciones en las tasas de suicidio con factores económicos, sociales, demográficos y culturales. En México, entre 1950 y 2008, el aumento de las tasas de suicidio de hombres jóvenes y ancianos fue atribuido a los cambios demográficos, las migraciones, el escaso acceso a la educación y la disminución del ingreso per cápita (57). En Australia se detectó un alto riesgo de suicidio en los grupos de las áreas geográficas que tenían condiciones socioeconómicas de nivel medio a bajo y altas tasas de desempleo $(58,59)$.

Los cambios observados en las tasas de suicidios pueden obedecer a que los individuos con riesgo de suicidio y los que se suicidan son particularmente sensibles a los cambios sociales, económicos y culturales de la sociedad en la que viven $(60,61)$. Por ejemplo, la teoría socio-ambiental de Durkheim predice altas tasas de suicidio en las sociedades con bajos niveles de integración social o regulación social (62), aunque se ha visto que las áreas con mayor privación tienen una menor tasa de suicidio (61). Por otra parte, cuando se consideran algunos indicadores sociales, económicos y demográficos del contexto, se encuentra que los suicidios varían según las condiciones sociales y económicas que primen en el momento.

En el caso de Colombia, el incremento de las tasas de suicidio entre 1979 y 1999-2000 puede deberse a que durante las últimas cinco décadas el país enfrentó una situación de violencia que se generó principalmente en las áreas rurales, donde aparecieron grupos armados al margen de la ley, cultivos ilícitos y narcotráfico, problemas que originaron el conflicto armado y la crisis económica, política y social. En la disputa por la tenencia de la tierra que se dio entre los distintos grupos que intervinieron, se presentaron extorsiones, cobro ilegal de impuestos, secuestros y expropiación de bienes que, finalmente, ocasionaron el desplazamiento de los habitantes del área rural (63). En 1979, el 36,1\% de la población habitaba el área rural y, en 2014, se redujo al 20,9\% (64). Por lo general, esta población ha sido pobre y con bajo nivel educativo y, por efecto del conflicto, se quedó sin tierra, sin oportunidades de empleo y sin medios de subsistencia (63). El desplazamiento forzoso, al que se aunó la migración masiva, produjo también consecuencias socioeconómicas en el sector urbano (63).

Entre 1999 y 2000, las tasas de suicidio mostraron tendencia a la reducción, como ocurrió en China entre 1987 y 2014, donde se observó que descendieron las tasas por sexo y por área en individuos de 65 y más años (65). Después del periodo 1999-2000 en el país se observó mejoría en algunos de los indicadores socioeconómicos que, en parte, podrían explicar el descenso de las tasas de suicidio. Entre el 2000 y el 2014, el producto interno bruto (PIB) aumentó el 44,7 \%; el desempleo cayó de 15,5 \%a 9,1\%; la inflación también descendió de 28,82 en 1979 a 3,66 en el 2014 (66).

Después de la crisis de 1999, el porcentaje de colombianos bajo la línea de pobreza disminuyó, de $50 \%$ en el 2002 pasó a $28 \%$ en el 2016. El porcentaje de pobres extremos bajó de 18 a $9 \%$ en el mismo periodo. La pobreza 
multidimensional cayó de 30 a $18 \%$ entre el 2010 y el 2016 (67). En el 2005, el índice de pobreza multidimensional fue de $39 \%$ en el área urbana y de $80 \%$ en la rural (68). En el 2014, este índice fue de $15,4 \%$ en las áreas urbanas y de $44,1 \%$ en las rurales. La tasa de desempleo descendió paulatinamente, en el 2002 era de 15,52 \% y en el 2014 se redujo a 9,38 \% (66). Del mismo modo, el índice de fecundidad descendió, en 1979 fue de 4,07 y en el 2014 de 1,9 (69).

Por otra parte, en el comportamiento de las tasas de suicidio no se debe olvidar el papel que tienen los factores relacionados con la familia y con la salud mental, y que probablemente han incidido en las diferencias entre las áreas urbanas y las rurales. Los fenómenos migratorios pudieron originar la separación de las familias, de los amigos y de los compañeros de trabajo, hecho que al producir estrés y afectación mental entre los que se quedaron, los pudo llevar al suicidio. No obstante, entre los que se quedaron quizá mejoró la calidad de vida, siempre y cuando el entorno no fuera adverso, y de esta manera, las tasas de suicidio fueron menores (70).

Con respecto a la atención de la enfermedad mental de la población, se han tenido avances. Entre 1970 y 1989, la atención estaba dirigida al tratamiento farmacológico. A partir de 1993, las escasas acciones en salud mental estaban reducidas a la intervención de pacientes en crisis, y a unas pocas actividades de prevención con acceso y cobertura limitada. Para el 2007, se propusieron acciones orientadas a la promoción de la salud mental, a la prevención de la violencia, el maltrato, la drogadicción y el suicidio, y al tratamiento de los trastornos mentales de mayor prevalencia (71).

A pesar de estos adelantos, en la población no ha existido culturalmente la costumbre de consultar a los profesionales de salud mental, lo cual probablemente se deba al estigma que sufren las personas con enfermedad mental. Al estigmatizarlas, no solo se impide que las personas busquen ayuda, sino que se exponen a un mayor riesgo de suicidio, ya que este puede parecer la mejor solución para ellas. Además, el suicidio es una fuente de estigma, pues cualquier persona con ideación suicida se considera débil, vergonzosa y egoísta, lo que impide que busquen tratamiento temprano en el proceso suicida (72).

Asimismo, la presencia de cualquier trastorno mental, como los depresivos y de ansiedad, y los rasgos limítrofes de personalidad, están asociados con la ideación suicida grave (73). En este contexto, según la encuesta de salud mental, en 2015 el 7,0 \% de los adultos que había pensado en suicidarse era del área urbana y el $5 \%$ de la rural. De los adultos que tenían plan suicida, el 2,4\% era del área urbana. De los adultos que intentaron suicidarse, el 2,6 $\%$ era del área urbana y el $2,4 \%$ de la rural (74). En cuanto al hogar urbano, se presentó diferencia en la ideación suicida, pues quienes refirieron no tener relación con el hogar urbano, tuvieron una mayor prevalencia en el grupo de ideación suicida (82,6 \%) que los que sí la tuvieron (76,9\%) (73).

En lo concerniente a los métodos empleados para el suicidio, el envenenamiento fue más frecuente en el área rural y el ahorcamiento en la urbana. Esto es similar a lo encontrado en otros países, especialmente en aquellos en desarrollo, donde el envenenamiento es el método más común de suicidio en las zonas rurales $(6,41)$.

En cuanto al método empleado para el suicidio, los hombres utilizan métodos más violentos, como las armas de fuego, el ahorcamiento, el salto desde lugares altos y el envenenamiento (por gas), mientras que las mujeres recurren a métodos menos agresivos, como el envenenamiento (sobredosis de 
sustancias psicotrópicas o venenos) o las armas cortantes (75). La selección del método se basa en su disponibilidad. En las áreas rurales, prevalece el envenenamiento debido al fácil acceso a los plaguicidas empleados en agricultura y, en las áreas urbanas, se prefieren las armas de fuego (76).

Los resultados presentados deben interpretarse con precaución, pues este análisis tiene limitaciones. El estudio recurrió a las definiciones de área urbana y rural utilizadas en el país, las que pueden diferir de las empleadas en otros lugares. También, se debe considerar la calidad de la información en su cobertura y su contenido. Es probable que haya problemas de subregistro y mala clasificación de los casos de suicidio, debido a que en su certificación se involucran aspectos médicos y legales (2).

La tendencia presentada señala el comportamiento del suicidio en Colombia. El riesgo de suicidio fue diferente entre áreas urbanas y rurales. Los hombres tuvieron mayor riesgo de suicidio en las dos áreas. Se observaron diferencias por grupos de edad y por métodos de suicidio. La tendencia mostró variaciones temporales y según el sexo, el grupo de edad y el método empleado, pero, en general, mostró una reducción en las tasas de los últimos años. El conocimiento del comportamiento del suicidio por áreas permite identificar grupos de alto riesgo para orientar mejor la adopción de decisiones en torno a su prevención.

\section{Referencias}

1. Neeleman J. Beyond risk theory: Suicidal behavior in its social and epidemiological context. Crisis. 2002;23:114-20. https://doi.org/10.1027//0227-5910.23.3.114

2. Organización Mundial de la Salud. Prevención del suicidio: un imperativo global. Prevencion del Suicidio. Washington, D.C.: Organización Panamericana de la Salud; 2014. p. 92.

3. World Health Organization. Suicide rates (per 100000 population) 2015. Fecha de consulta: 12 de febrero de 2018. Disponible en: http://www.who.int/gho/mental health/suicide rates/en/

4. Instituto Nacional de Salud. Mortalidad 1998-2011 y situación de salud en los municipios de frontera terrestre en Colombia. Bogotá: Instituto Nacional de Salud; 2013. p. 237.

5. Stack S. Suicide: A decade review of the sociological literature. Deviant Behav. 1982;4:4166. https://doi.org/10.1016/j.jadohealth.2003.10.007

6. Hirsch JK. A review of the literature on rural suicide: Risk and protective factors, incidence, and prevention. Crisis. 2006;27:189-99. https://doi.org/10.1027/0227-5910.27.4.189

7. Phillips MR, Yang G, Zhang Y, Wang L, Ji H. Risk factors for suicide in China: A national case-control psychological autopsy study. Lancet. 2002;360:1728-36. https://doi.org/10.1016/S0140-6736(02)11681-3

8. Saunderson T, Haynes R, Langford IH. Urban-rural variations in suicides and undetermined deaths in England and Wales. J Public Health Med. 1998;20:261-7.

9. Morrell S, Taylor R, Slaytor E, Ford P. Urban and rural suicide differentials in migrants and the Australian-Born, New South Wales, Australia 1985-1994. Soc Sci Med. 1999;49:81-91. https://doi.org/10.1016/S0277-9536(99)00083-0

10. Dudley M, Kelk N, Florio T, Howard J, Waters B. Suicide among young Australians, 19641993: An interstate comparison of metropolitan and rural trends. Med J Aust. 1998;169:77-80.

11. Razvodovsky Y, Stickley A. Suicide in urban and rural regions of Belarus, 1990-2005. Public Health. 2009;123:27-31. https://doi.org/10.1016/j.puhe.2008.10.003

12. Cheong KS, Choi MH, Cho BM, Yoon TH, Kim CH, Kim YM, et al. Suicide rate differences by sex, age, and urbanicity, and related regional factors in Korea. J Prev Med Public Health. 2012;45:70-7. https://doi.org/10.3961/jpmph.2012.45.2.70

13. Ryu J. Regional differentials in age group specific suicide rate in South Korea 2005-2010. Korea J Popul Stud. 2008;31:21-44.

14. Patel V, Ramasundarahettige C, Vijayakumar L, Thakur JS, Gajalakshmi V, Gururaj G, et al. Suicide mortality in India: A nationally representative survey. Lancet. 2012;379:2343-51. https://doi.org/10.1016/S0140-6736(12)60606-0 
15. Chen YY, Kwok CL, Yip PS, Wu KC. A test of the substitution hypothesis: An analysis of urban and rural trends in solid/liquid poisoning suicides in Taiwan. Soc Sci Med. 2013;96:4551. https://doi.org/10.1016/j.socscimed.2013.06.031

16. Zhang L, Li Z, Li X, Zhang J, Zheng L, Jiang C, et al. Study on the trend and disease burden of injury deaths in Chinese population, 2004-2010. PLoS One. 2014;9:2004-10. https://doi.org/10.1371/journal.pone.0085319

17. Najafi F, Hasanzadeh J, Moradinazar M, Faramarzi H, Nematollahi A. An epidemiological survey of the suicide incidence trends in the Southwest Iran: 2004-2009. Int J Health Policy Manag. 2013;1:219-22. https://doi.org/10.15171/ijhpm.2013.40

18. Wilkinson D, Gunnell D. Youth suicide trends in Australian metropolitan and non-metropolitan areas, 1988-1997. Aust N Z J Psychiatry. 2000;34:822-8. https://doi.org/10.1080/j.1440-1614.2000.00812.x

19. Caldwell TM, Jorm AF, Dear KB. Suicide and mental health in rural, remote and metropolitan areas in Australia. Med J Aust. 2004;181(Suppl.7):10-4.

20. Dudley M, Kelk N, Florio T, Howard J, Waters B, Haski C, et al. Suicide among young rural Australians 1964-1993: A comparison with metropolitan trends. Soc Psychiatry Psychiatr Epidemiol. 1997;32:251-60.

21. Cha ES, Khang YH, Lee WJ. Mortality from and incidence of pesticide poisoning in South Korea: Findings from national death and health utilization data between 2006 and 2010. PLoS One. 2014;9:e95299. https://doi.org/10.1371/journal.pone.0095299

22. Singh GK, Siahpush M. Increasing rural -urban gradients in US suicide. Am J Public Health. 2002;92:1161-7.

23. Pearce J, Barnett R, Jones I. Have urban/rural inequalities in suicide in New Zealand grown during the period 1980-2001? Soc Sci Med. 2007;65:1807-19. https://doi.org/10.1016/j.socscimed.2007.05.044

24. Kapusta ND, Zorman A, Etzersdorfer E, Ponocny-Seliger E, Jandl-Jager E, Sonneck G. Rural-urban differences in Austrian suicides. Soc Psychiatry Psychiatr Epidemiol. 2008;43:311-8. https://doi.org/10.1007/s00127-008-0317-1

25. Levin KA, Leyland AH. Urban/rural inequalities in suicide in Scotland, 1981-1999. Soc Sci Med. 2005;60:2877-90. https://doi.org/10.1016/i.socscimed.2004.11.025

26. Eddleston M, Sudarshan K, Senthilkumaran M, Reginald K, Karalliedde L, Senarathna L, et al. Patterns of hospital transfer for self-poisoned patients in rural Sri Lanka: Implications for estimating the incidence of self-poisoning in the developing world. Bull World Health Organ. 2006;84:276-82.

27. Gunnell D, Eddleston M, Phillips MR, Konradsen F. The global distribution of fatal pesticide self-poisoning: Systematic review. BMC Public Health. 2007;7:357. https://doi.org/10.1186/1471-2458-7-357

28. Chang SS, Chen YY, Yip PS, Lee WJ, Hagihara A, Gunnell D. Regional changes in charcoal-burning suicide rates in East/Southeast Asia from 1995 to 2011: A time trend analysis. PLoS Med. 2014;11:e1001622. https://doi.org/10.1371/journal.pmed.1001622

29. Tuesca R, Navarro E. Factores de riesgo asociados al suicidio e intento de suicidio. Salud Uninorte. 2003;17:19-28.

30. Franco SA, Gutiérrez ML, Sarmiento J, Cuspoca D, Tatis J, Castillejo A, et al. Suicidio en estudiantes universitarios en Bogotá, Colombia, 2004-2014. Cien Saude Colet. 2017;22:269-78. https://doi.org/10.1590/1413-81232017221.22452015

31. Cendales R, Vanegas C, Fierro M, Córdoba R, Olarte A. Tendencias del suicidio en Colombia, 1985-2002. Rev Panam Salud Pública. 2007;22:231-8.

32. Cardona D, Medina-Pérez A, Cardona D. Characterisation of suicide in Colombia. Rev Colomb Psiquiatr. 2016;45:170-7. https://doi.org/10.1016/j.rcp.2015.10.002

33. Gómez-Restrepo C, Rodríguez N, C. de Romero L, Pinilla C, López E, Díaz-Granados N, et al. Suicidio y lesiones autoinfligidas, Colombia, 1973-1996. Rev Colomb Psiquiatr. 2002;2:123-36.

34. Ortega PA, Manrique RD, Tovilla CA, López C, Cuartas JM. Clinical and epidemiological characteristics of suicides committed in Medellin, Colombia. Rev Colomb Psiquiatr. 2014;43:106-12. https://doi.org/10.1016/.rcp.2014.02.006

35. Palacio-Acosta C, García-Valencia J, Diago-García J, Zapata C, Ortiz-Tobón J, López-Calle $\mathrm{G}$, et al. Characteristics of people committing suicide in Medellín, Colombia. Rev Salud Pública (Bogotá). 2005;7:243-53. 
36. Sánchez R, Orejarena S, Guzmán Y. Características de los suicidas en Bogotá: 1985-2000. Rev Salud Pública (Bogotá). 2004;6:217-34.

37. Torres L. ¿Existe una relación entre rendimiento académico y muerte por suicidio?: estudio retrospectivo en la Universidad de los Andes, 1992-2002. Bogotá: Universidad de los Andes; 2003. p. 48.

38. Dávila CA, Pardo AM, Pardo AM. Mortalidad por suicidios en Colombia y México: tendencias e impacto entre 2000 y 2013. Biomédica. 2016;36:415-22. https://doi.org/10.7705/ biomedica.v36i3.3224

39. Cardona D, Segura ÁM, Espinosa A, Segura A. Homicidios y suicidios en jóvenes de 15 a 24 años, Colombia, 1998-2008. Biomédica. 2013;33:574-86. https://doi.org/10.7705/biomedica.v33i4.848

40. Campo-Arias A, Herazo E. Asociación entre desigualdad y tasa de suicidio en Colombia (1994-2013). Rev Colomb Psiquiatr. 2015;44:28-32.

41. García J, Montoya GJ, López CA, López MC, Montoya P, Arango JC, et al. Características de los suicidios de áreas rurales y urbanas de Antioquia, Colombia. Rev Colomb Psiquiatr. 2011;40:199-214. https://doi.org/10.1016/S0034-7450(14)60118-9

42. Departamento Administrativo Nacional de Estadística. Estadísticas vitales nacimientos y defunciones. Fecha de consulta: 12 de febrero de 2018. Disponible en: http://www.dane.gov.co/index.php/estadisticas-por-tema/salud/nacimientos-y-defunciones

43. Organización Panamericana de la Salud. Manual de la clasificación estadística internacional de enfermedades, traumatismos y causas de defunción. Novena revisión. Washington, D.C.: Organización Panamericana de la Salud; 1978.

44. Organización Panamericana de la Salud. Manual de la clasificación estadística internacional de enfermedades, traumatismos y causas de defunción. Décima revisión. Washington, D.C.: Organización Panamericana de la Salud; 1995.

45. Departamento Administrativo Nacional de Estadística. Series de población. Fecha de consulta: 12 de febrero de 2018. Disponible en: https://www.dane.gov.co/index.php/ estadisticas-por-tema/demografia-y-poblacion/series-de-poblacion

46. Departamento Administrativo Nacional de Estadística. Conceptos básicos. Pagina oficial del DANE. 1994. Fecha de consulta: 12 de febrero de 2018. Disponible en: http://www.dane. gov.co/files/inf geo/4Ge ConceptosBasicos.pdf

47. Agresti A. Categorical data analysis. Second edition. New Jersey: Wiley Series in Probability and Statistics; 2002. p. 710.

48. Kim HJ, Fay MP, Feuer EJ, Midthune DN. Permutation tests for joinpoint regression with applications to cancer rates. Stat Med. 2000;19:335-51.

49. National Cancer Institute. Methodology for characterizing trends. Fecha de consulta: 12 de febrero de 2018. Disponible en: https://progressreport.cancer.gov/methodology

50. National Cancer Institute. Joinpoint trend analysis software, version 4.5.0.1. Fecha de consulta: 15 de junio de 2018. Disponible en: https://surveillance.cancer.gov/joinpoint/

51. Kanchan T, Menon A, Menezes RG. Methods of choice in completed suicides: Gender differences and review of literature. J Forensic Sci. 2009;54:938-42. https://doi.org/1 10.1111/j.1556-4029.2009.01054.x

52. Canetto SS, Sakinofsky I. The gender paradox in suicide. Suicide Life Threat Behav. 1998;28:1-23. https://doi.org/10.1111/j.1943-278X.1998.tb00622.x

53. Breuer C. Unemployment and suicide mortality: Evidence from regional panel data in Europe. Health Econ. 2015;24:936-50. https://doi.org/10.1002/hec.3073

54. Koo J, Cox WM. An economic interpretation of suicide cycles in Japan. Contemp Econ Policy. 2008;26:162-74. https://doi.org/10.1111/j.1465-7287.2007.00042.x

55. Pérez SA. Factores de riesgo suicida en el anciano. Cien Saude Colet. 2012;17:2011-6. https://doi.org/10.1590/S1413-81232012000800012

56. Yip PS, Callanan C, Yuen HP. Urban/rural and gender differentials in suicide rates: East and West. J Affect Disord. 2000;57:99-106. https://doi.org/10.1016/S0165-0327(99)00058-0

57. Hernández-Bringas H, Flores-Arenales R. El suicidio en México. Papeles de Población. 2011;17:69-101. 
58. Qi X, Hu W, Page A, Tong S. Spatial clusters of suicide in Australia. BMC Psychiatry. 2012;12:86. https://doi.org/10.1186/1471-244X-12-86

59. Qi X, Hu W, Mengersen K, Tong S. Socio-environmental drivers and suicide in Australia: Bayesian spatial analysis. BMC Public Health. 2014;14:1-10. https://doi.org/10.1186/1471-2458-14-681

60. Goldney RD, Schioldann JA, Dunn KI. Suicide research before Durkheim. Health History. 2018;10:73-93.

61. Milner A, Hjelmeland H, Arensman E, Leo D. Social-environmental factors and suicide mortality: A narrative review of over 200 articles. Sociol Mind. 2013;03:137-48. https://doi.org/10.4236/sm.2013.32021

62. Durkheim E. El suicidio. Madrid: Editorial Reus S. A.; 1928. p. 450.

63. Pérez E, Pérez M. El sector rural en Colombia y su crisis actual. Cuadernos de Desarrollo Rural. 2002;48:35-58.

64. Comisión Económica para América Latina y el Caribe, CEPAL. Estimaciones y proyecciones de población total, urbana y rural, y económicamente activa. Colombia. Estimaciones y proyecciones de población urbana y población rural según sexo y grupos quinquenales de edad. 2017. Fecha de consulta: 12 de febrero de 2018. Disponible en: https:/www.cepal.org/es/temas/proyeccionesdemograficas/estimaciones-proyecciones-poblacion-total-urbana-rural-economicamente-activa

65. Zhong BL, Chiu HF, Conwell Y. Elderly suicide trends in the context of transforming China, 1987-2014. Sci Rep. 2016;6:37724. https://doi.org/10.1038/srep37724

66. Banco de la República. Información recopilada y calculada por el Departamento Técnico y de Información Económica del Banco de la República. Bogotá D.C.; 2018. Fecha de consulta: 12 de febrero de 2018. Disponible en: http://web.sirhuila.gov.co/images/sirhuila/ SIR 2018/BOLETINES/BANCO DE LA REPUBLICA/Boletn-indicadores-econmicosBanco-de-la-Repblica-23-de-abril-de-2018.pdf

67. DANE. Pobreza monetaria y multidimensional en Colombia 2016. Anexos [Internet]. Fecha de consulta: 26 de febrero de 2018. Disponible en: https://www.dane.gov.co/index.php/ estadisticas-por-tema/pobreza-y-condiciones-de-vida/pobreza-y-desigualdad/pobrezamonetaria-y-multidimensional-en-colombia-2016

68. DANE. Indice de pobreza multimodal por municipio y departamento 2005 incidencias y privaciones. Bogotá; 2005. Fecha de consulta: 12 de febrero de 2018. Disponible en: https://formularios.dane.gov.co/Anda_4_1/index.php/catalog/392

69. Expansion.com/Datosmacro.com. Colombia natalidad. Fecha de consulta: 26 de febrero de 2018. Disponible en: https://www. datosmacro.com/demografia/natalidad/colombia

70. Carcach C. A spatio-temporal analysis of suicide in El Salvador. BMC Public Health. 2017;17:339. https://doi.org/10.1186/s12889-017-4251-6

71. Comité de Políticas, Asociación Colombiana de Psiquiatría. Política Nacional del Campo de la Salud Mental. Documento-propuesta para discusión y acuerdos. Bogotá: Ministerio de la Protección Social; 2007.

72. Pompili M, Mancinelli I, Tatarelli R. Stigma as a cause of suicide. Br J Psychiatry. 2003;183:173-4. https://doi.org/10.1192/S0007125000162774

73. Arenas A, Gómez-Restrepo C, Rondón M. Factores asociados a la conducta suicida en Colombia. Resultados de la Encuesta Nacional de Salud Mental 2015. Rev Colomb Psiquiatr. 2016;45(Supl.1):68-75.

74. Ministerio de Salud y Protección Social, Colciencias. Encuesta Nacional de Salud Mental, 2015. Bogotá: Minsalud; 2015. p. 1-242.

75. García de Jalon E, Peralta V. Suicidio y riesgo de suicidio. Ann Psychol Sis San Navarra. 2002;25:87-96

76. Cano-Montalbán I, Quevedo-Blasco R. Sociodemographic variables most associated with suicidal behaviour and suicide methods in Europe and America. A systematic review. European Journal of Psychology Applied to Legal Context. 2018;10:15-25. https://doi.org/10.5093/ejpalc2018a2 